\title{
Correlation Between Oxygen Vacancy, Microstrain, and Cation Distribution in Lithium-Excess Layered Oxides During the First Electrochemical Cycle
}

\author{
Christopher R. Fell, ${ }^{\dagger, \|}$ Danna Qian, ${ }^{\S}$ Kyler J. Carroll, ${ }^{\S}$ Miaofang Chi, ${ }^{\ddagger}$ Jacob L. Jones, ${ }^{\dagger}$ \\ and Ying Shirley Meng $*, \dagger, \S$ \\ ${ }^{\dagger}$ Department of Materials Science and Engineering, University of Florida, Gainesville, Florida 32611, United States \\ ${ }^{\ddagger}$ Materials Science and Technology Division, Oak Ridge National Laboratory, Oak Ridge, Tennessee 37831, United States \\ ${ }^{\S}$ Department of NanoEngineering, University of California San Diego, La Jolla, California 92037, United States
}

\section{Supporting Information}

ABSTRACT: Dynamic structural changes during the first electrochemical charge and discharge cycle in the Li-excess layered oxide compound, $\mathrm{Li}\left[\mathrm{Li}_{1 / 5} \mathrm{Ni}_{1 / 5} \mathrm{Mn}_{3 / 5}\right] \mathrm{O}_{2}$, are studied with synchrotron X-ray diffraction (SXRD), aberration corrected scanning transmission electron microscopy (a-S/TEM), and electron energy loss spectroscopy (EELS). At different states of charge, we carefully examined the crystal structures and electronic structures within the bulk and have found that increased microstrain is accompanied with the cation migration and a second phase formation which occurs during the first cycle voltage plateau as well as into the beginning of the discharge cycle. The evidence indicates that the oxygen vacancy formation and activation may facilitate cation migration and results in the formation of a second phase. The EELS results reveal a $\mathrm{Mn}$ valence change from 4+ to 3+ upon oxygen vacancy formation and recovers back to $4+$ at the discharge. The oxygen vacancy formation and activation at the partially

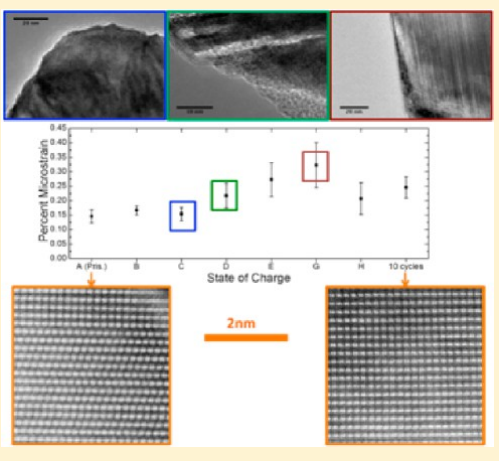
delithiated state leads to the generation of several crystal defects which are observed in TEM. Identification of the correlation between microstrain and oxygen vacancy formation during the first electrochemical cycle clarifies the complex intercalation mechanisms that accounts for the anomalous capacities exceeding $200 \mathrm{mAh} / \mathrm{g}$ in the Li-excess layered oxide compounds.

KEYWORDS: lithium ion batteries, lithium-excess layered oxide, cathode materials, oxygen vacancy, microstrain

\section{INTRODUCTION}

Rechargeable lithium ion batteries are a potential candidate for the use as the primary onboard storage technology in plug-in hybrid electric vehicles (PHEVs) or electric vehicles (EVs). Specifically, the layered lithium-excess transition metal oxides, $\mathrm{Li}\left[\mathrm{Ni}_{x} \mathrm{Li}_{1 / 3-2 x / 3} \mathrm{Mn}_{2 / 3-x / 3}\right] \mathrm{O}_{2}(0<x<1 / 2)$, are of great interest as a new generation of positive electrode materials since they deliver higher reversible capacity exceeding $250 \mathrm{mAh} / \mathrm{g}$ at a lower cost compared with both $\mathrm{LiCoO}_{2}$ and $\mathrm{LiNiO}_{2}$ based materials. ${ }^{1}$ The structure of the $\mathrm{Li}\left[\mathrm{Ni}_{x} \mathrm{Li}_{1 / 3-2 x / 3} \mathrm{Mn}_{2 / 3-x / 3}\right] \mathrm{O}_{2}$ system is controversial and has been identified as a solid solution or as a two phase mixture of the two end members, $\mathrm{Li}_{2} \mathrm{MnO}_{3}$ and $\mathrm{Li}\left[\mathrm{Ni}_{1 / 2} \mathrm{Mn}_{1 / 2}\right] \mathrm{O}_{2}{ }^{2,3}$ The structure differs from conventional layered transition metal oxides because the excess lithium is positioned in the transition metal layer and the excess $\mathrm{Li}^{+}$ions form an ordered pattern with the transition metal (TM) ions in the TM layer. ${ }^{4,5}$ Previous research has identified that the initial capacity, during the sloping region up to $4.40 \mathrm{~V}$, originates from the oxidation of $\mathrm{Ni}^{2+}$ to $\mathrm{Ni}^{4+}$. ${ }^{4}$ At $4.45 \mathrm{~V}$, a voltage plateau region appears which drastically improves the capacity; however, it is associated with a large irreversible capacity on the first discharge of 40 to $100 \mathrm{mAh} / \mathrm{g} .{ }^{8-10}$ It has been previously identified that the lithium chemical diffusion coefficient is significantly lower at the plateau region. ${ }^{11}$ Recently $\mathrm{Li}$ et al. reported that the capacity originating from the high voltage plateau is strongly dependent on the charging current indicating slow kinetics. ${ }^{12}$

Considerable research has been devoted to address the irreversible capacity losses as well as the poor rate capability by understanding the mechanisms associated with the first electrochemical charge/discharge cycle. Researchers have proposed simultaneous $\mathrm{Li}^{+}$and $\mathrm{O}^{2-}$ diffusion creating oxygen vacancies and surface transition metal migration. ${ }^{7,8,13-15}$ These structural rearrangements are believed to happen mainly during the first electrochemical cycle since the voltage plateau does not appear in subsequent cycles. For oxygen diffusion to occur, oxygen must overcome the activation energy barrier, a process that has been widely studied in ionic conductors such as solid oxide fuel cell electrolytes. ${ }^{16-20}$ The diffusion mechanism can be explained by interstitialcy, interstitials, and vacancy

Received: January 2, 2013

Revised: $\quad$ March 20, 2013

Published: March 21, 2013 
mechanisms depending on point defects in the system. The introduction of strain at or near interfaces has a powerful effect on the ionic diffusion properties of ceramics; ${ }^{21,22}$ however, the relation of lattice strain and ionic transport in the lithium excess layered oxides is not well understood. Lattice strains first elastically stretch the cation-oxygen bond, effectively weakening the bond and decreasing the migration barrier leading to exponential increases in oxygen diffusivity. ${ }^{16,17,23,24}$ During electrochemical cycling of the layered lithium-excess series of materials, significant cationic rearrangement occurs. It has also been shown that following electrochemical cycling the superlattice peaks disappear indicating a loss of $\mathrm{Li} / \mathrm{Mn}$ honeycomb-type ordering. ${ }^{7,15,25}$ During electrochemical cycling, the material shows volume expansion and significant shifts in the $c / a$ lattice ratio. ${ }^{8,11,15,26}$ At the surface of the material, changes in TM oxidation state and local environments as well as possible material loss have been reported. ${ }^{5,9,14,26}$ Recent research shows the formation of a defect-like spinel phase that may contribute to the first cycle irreversible capacity and poor rate capability. ${ }^{26,27} \mathrm{We}$ hypothesize that a combination of these changes during the electrochemical cycling provides the defect sites that enable oxygen mobility at ambient temperature.

It is crucial to understand how defects are generated and to quantify the microstrain associated with these defects. In this work, we carried out a detailed study using synchrotron X-ray diffraction (SXRD), aberration corrected scanning transmission electron microscopy (a/STEM), and electron energy loss spectroscopy (EELS) combined with electrochemical testing on $\mathrm{Li}\left[\mathrm{Li}_{1 / 5} \mathrm{Ni}_{1 / 5} \mathrm{Mn}_{3 / 5}\right] \mathrm{O}_{2}$ compounds during the first electrochemical cycle at different states of charge to identify the structural changes. Our research findings provide new insights into the complex intercalation mechanisms and how they influence dynamic structural changes in the $\mathrm{Li}$-excess layered oxide compound, $\mathrm{Li}\left[\mathrm{Li}_{1 / 5} \mathrm{Ni}_{1 / 5} \mathrm{Mn}_{3 / 5}\right] \mathrm{O}_{2}$, during the first electrochemical charge and discharge cycle.

\section{EXPERIMENTAL SECTION}

Synthesis. A coprecipitation technique was used for the synthesis of the materials which was previously described. ${ }^{11}$ Transition metal nitrates, $\mathrm{Ni}\left(\mathrm{NO}_{3}\right)_{2} \cdot 6 \mathrm{H}_{2} \mathrm{O}$ (Fisher) and $\mathrm{Mn}\left(\mathrm{NO}_{3}\right)_{2} \cdot 4 \mathrm{H}_{2} \mathrm{O}$ (Fisher), were titrated into a stoichiometric $\mathrm{LiOH} \cdot \mathrm{H}_{2} \mathrm{O}$ (Fisher) solution for a duration of two hours. The coprecipitated transition metal hydroxides were then filtered using a vacuum filter and washed three times with deionized water. The collected transition metal hydroxides were dried in an oven at $180{ }^{\circ} \mathrm{C}$ for $10 \mathrm{~h}$ in air. The dried transition metal precursors were mixed with a stoichiometric amount of $\mathrm{LiOH} \cdot \mathrm{H}_{2} \mathrm{O}$ corresponding to the amount of $\mathrm{M}(\mathrm{OH})_{2}$ from the coprecipitation step. This mixture was ground for $30 \mathrm{~min}$ to ensure adequate mixing and then placed into a furnace at $480^{\circ} \mathrm{C}$ for $12 \mathrm{~h}$. The precalcinated powders were prepared as a pellet for high temperature sintering. These samples were then calcinated at $1000^{\circ} \mathrm{C}$ for $12 \mathrm{~h}$ in air. Samples were brought back to room temperature by furnace cooling.

Electrochemistry. Electrochemical properties were measured on an Arbin battery cycler in galvanostatic mode between 4.8 and $2.0 \mathrm{~V}$. Cathodes were prepared by mixing the active material $\mathrm{Li}$ $\left[\mathrm{Li}_{1 / 5} \mathrm{Ni}_{1 / 5} \mathrm{Mn}_{3 / 5}\right] \mathrm{O}_{2}$ with 10 wt \% Super P carbon (TIMCAL) and $10 \mathrm{wt} \%$ poly(vinylidene fluoride) (PVDF) in $\mathrm{N}$-methylpyrrolidone (NMP) solution. The slurry was cast onto an $\mathrm{Al}$ foil using a doctor blade and dried in a vacuum oven overnight at $80{ }^{\circ} \mathrm{C}$. The electrode discs were punched and dried again at $80{ }^{\circ} \mathrm{C}$ for $6 \mathrm{~h}$ before storing them in an argon filled glovebox $\left(\mathrm{H}_{2} \mathrm{O}\right.$ level $\left.<1 \mathrm{ppm}\right) .2016$ type coin cells were used to electrochemically cycle $\mathrm{Li}\left[\mathrm{Li}_{1 / 5} \mathrm{Ni}_{1 / 5} \mathrm{Mn}_{3 / 5}\right] \mathrm{O}_{2}$ to different states of charge during the first electrochemical cycle seen in Supporting Information Figure S1. The batteries were prepared in the same Argon filled glovebox using lithium metal ribbon as an anode and a $1 \mathrm{M} \mathrm{LiPF} 6$ in a 1:1 ethylene carbonate/dimethyl carbonate (EC:DMC) electrolyte solution (Novolyte). Celgard model C480 separators (Celgard Inc., USA) were used as the separator.

The intermittently cycled samples were recovered by disassembling cycled batteries in the same argon-filled glovebox. The cathode was washed by submerging in acetonitrile 3 times and then allowed to dry in argon atmosphere overnight. For TEM samples, the powders were suspended on a copper grid with lacey carbon. The approximate time of sample exposed to air (from a sealed environment to the microscope column) is less than $5 \mathrm{~s}$.

Structural Characterization. Synchrotron $X$-ray Diffraction. Powder X-ray diffraction patterns were taken using synchrotron Xray diffraction at the Advanced Photon Source (APS) at Argonne National Laboratory (ANL) on beamline 11-BM $(E=30 \mathrm{keV}, \lambda=$ $0.413225 \AA$ A). All samples were hermetically sealed in $1.0 \mathrm{~mm}$ Kapton capillary to minimize air exposure. The beamline uses a sagittally focused X-ray beam with a high precision diffractometer circle and perfect $\mathrm{Si}(111)$ crystal analyzer detection for high sensitivity and resolution. Instrumental resolution at high $Q$ is better than $\Delta Q / Q \approx 2$ $\times 10^{-4}$, with a typical $2 \theta$ step size of $0.001^{\circ}$ at $30 \mathrm{keV}$. XRD data analysis was carried out by Rietveld refinement methods using FullProf and GSAS/EXPGUI software. ${ }^{28-30}$ Crystal structure refinement parameters included $2 \theta$, zero offset, intensity, scale factor, lattice parameters, isotropic atomic positions, atomic displacement parameters, and cation site occupancies. The refinements led to superior convergence factors when refined using the Thompson, Cox, and Hastings pseudo-Voigt function ${ }^{31}$ with axial divergence symmetry described by the Finger, Cox, and Jephcoat model. ${ }^{32}$

a-STEM/EELS. Electron microscopy work was carried out on a Cscorrected FEI Titan 80/300-kV TEM/STEM microscope equipped with a Gatan Image Filter Quantum-865. All STEM images and EELS spectra were acquired at $300 \mathrm{KV}$ and with a beam size of $\sim 0.7 \AA$. EELS spectra shown in this work were acquired from a square area of $\sim 0.5 \times$ $0.5 \mathrm{~nm}$ with an acquisition time of $3 \mathrm{~s}$ and a collection angle of 35 mrad. HAADF images were obtained with a convergence angle of 30 mrad and a large inner collection angle of $65 \mathrm{mrad}$. Images acquired by an HAADF detector with a small convergence angle and a relatively large inner collection angle are also called "Z-contrast" images, where the contrast is proportional to $Z^{1.7} \cdot 33,34$ Atomic resolution Z-contrast images can be used to differentiate elements and provide atomicstructural information based on the contrast of the atomic columns.

To minimize possible electron beam irradiation effects, EELS and HAADF figures presented in this work were acquired from areas without prebeam irradiation. $\mathrm{Mn}_{3}$ to $\mathrm{L}_{2}$ intensity ratio analysis was done by averaging over 8 to 12 spectra using the method described by Wang et al. ${ }^{35}$

\section{RESULTS}

Electrochemical measurements of $\mathrm{Li}\left[\mathrm{Li}_{1 / 5} \mathrm{Ni}_{1 / 5} \mathrm{Mn}_{3 / 5}\right] \mathrm{O}_{2}$ electrodes were performed in lithium half-cells. Figure S1 (Supporting Information) displays the first electrochemical charge/discharge curves between 4.8 and $2.0 \mathrm{~V}$ (at a rate of 10 $\mathrm{mA} / \mathrm{g}$ ) for the $\mathrm{Li}\left[\mathrm{Li}_{1 / 5} \mathrm{Ni}_{1 / 5} \mathrm{Mn}_{3 / 5}\right] \mathrm{O}_{2}$ electrode. The lettered points along the curves indicate states of charge, which were prepared for the various characterization techniques. Supporting Information Table S2 indicates the voltages, capacities and corresponding $\mathrm{Li}$ remaining in the material assuming all of the electrochemical capacity corresponds to $\mathrm{Li}^{+}$removal from or insertion into the material.

3.1. Synchrotron X-ray Diffraction. High resolution synchrotron X-ray diffraction was analyzed for detailed crystal structure evolution during the first electrochemical cycle for the $\mathrm{Li}\left[\mathrm{Ni}_{1 / 5} \mathrm{Li}_{1 / 5} \mathrm{Mn}_{3 / 5}\right] \mathrm{O}_{2}$ electrode material (Figure 1). The detailed fitting plots and refinement results are in Figure S3 and Table 1. Table 2 compares the Rietveld reliability factors for different states of charge for respective models. 

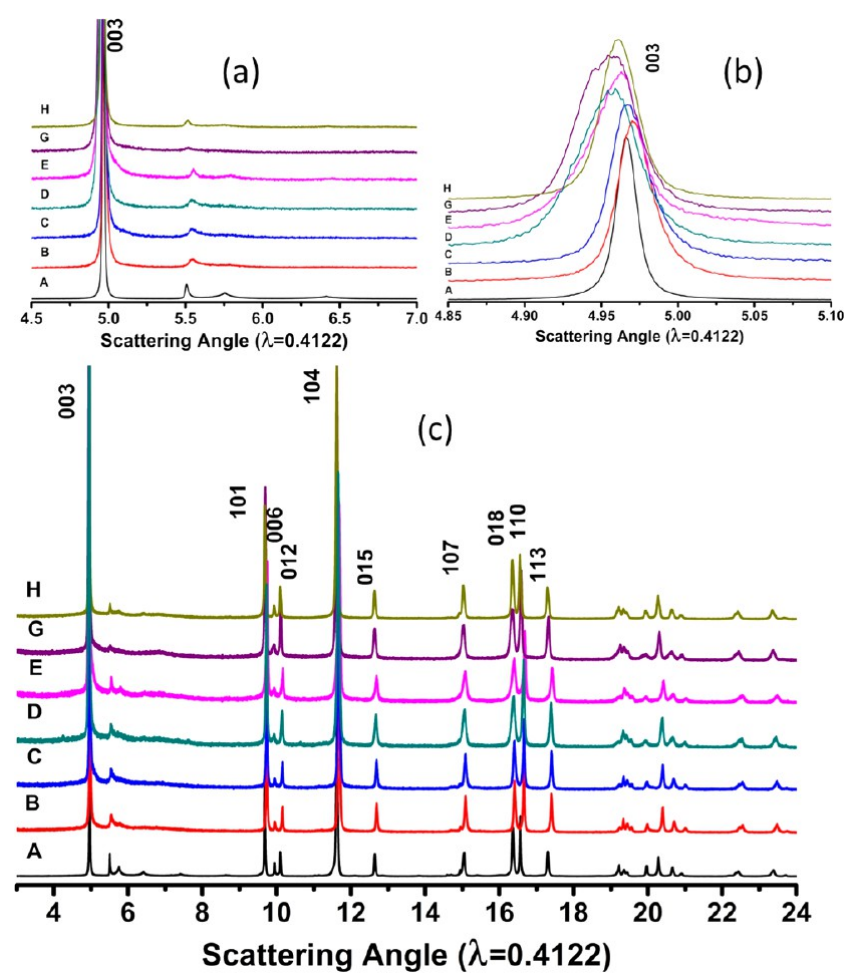

Figure 1. Synchrotron X-ray diffraction (XRD) patterns collected from different states of charge during the first electrochemical charge/ discharge cycle.

The X-ray diffraction pattern of the pristine material (A in Figure S1) was reproduced from ref $27 .{ }^{26}$ Rietveld refinement of the pristine material indicates the typical well-layered phase with $3 \% \mathrm{Li} / \mathrm{Ni}$ mixing. After charging the material to $4.40 \mathrm{~V}$, during the sloping region where $\mathrm{Ni}^{2+}$ is oxidized to $\mathrm{Ni}^{4+}$ during $\mathrm{Li}$ extraction, it is clear from XRD that the superlattice peaks remain visible and the transition metal ordering remains intact, as was previously shown from literature. ${ }^{8}$ Rietveld refinement at this position ( $\mathrm{B}$ in Figure $\mathrm{S} 1$ ) confirms that the material maintains the well-layered phase while the amount of $\mathrm{Li} / \mathrm{Ni}$ mixing decreases slightly. The $a$ lattice parameter decreases by $0.0089 \AA$ while the $c$ lattice parameter increases by $0.032 \AA$ from the pristine material (Table 2). This trend is consistent with the oxidation of $\mathrm{Ni}^{2+}$ to $\mathrm{Ni}^{4+}$, during which the ionic radius decreases from $0.69 \AA$ to $0.48 \AA$ inducing a contraction in the overall lattice. ${ }^{36}$ The refinement also confirms that the material remains a single layered phase with no obvious phase transformations occurring during the sloping region (comparison shown in Table 2).

After the sloping region (4.45 V, C in Figure S1), Rietveld refinement supports a single layered phase. The addition of tetrahedral $\mathrm{Li}^{+}$to the single phase model improved the Rietveld reliability factors $R_{\mathrm{b}}$ and $R_{\mathrm{wp}}$ to 10.73 and 4.93 . In addition, the amount of $\mathrm{Li} / \mathrm{Ni}$ mixing continues to decrease while the $a$ and $c$ lattice parameters continue to decrease and increase, respectively. This indicates that slightly after the sloping region, $\mathrm{Ni}^{2+}$ is still being oxidized to $\mathrm{Ni}^{4+}$, which is also confirmed by X-ray absorption spectroscopy (XAS) results (Figure S4). Rietveld refinement results of the XRD pattern cycled to this point provides the first evidence of tetrahedral $\mathrm{Li}^{+}$formation. This result is consistent with our previous findings that tetrahedral $\mathrm{Li}^{+}$ions are more energetically favorable to form in the middle of the first charge cycle. ${ }^{26}$
Table 1. Rietveld Refinement Results for $\mathrm{Li}\left[\mathrm{Li}_{1 / 5} \mathrm{Ni}_{1 / 5} \mathrm{Mn}_{3 / 5}\right] \mathrm{O}_{2}$ at Different States of Charge during the First Electrochemical Cycling Using One or Two Independent $R \overline{3} m$ Phases

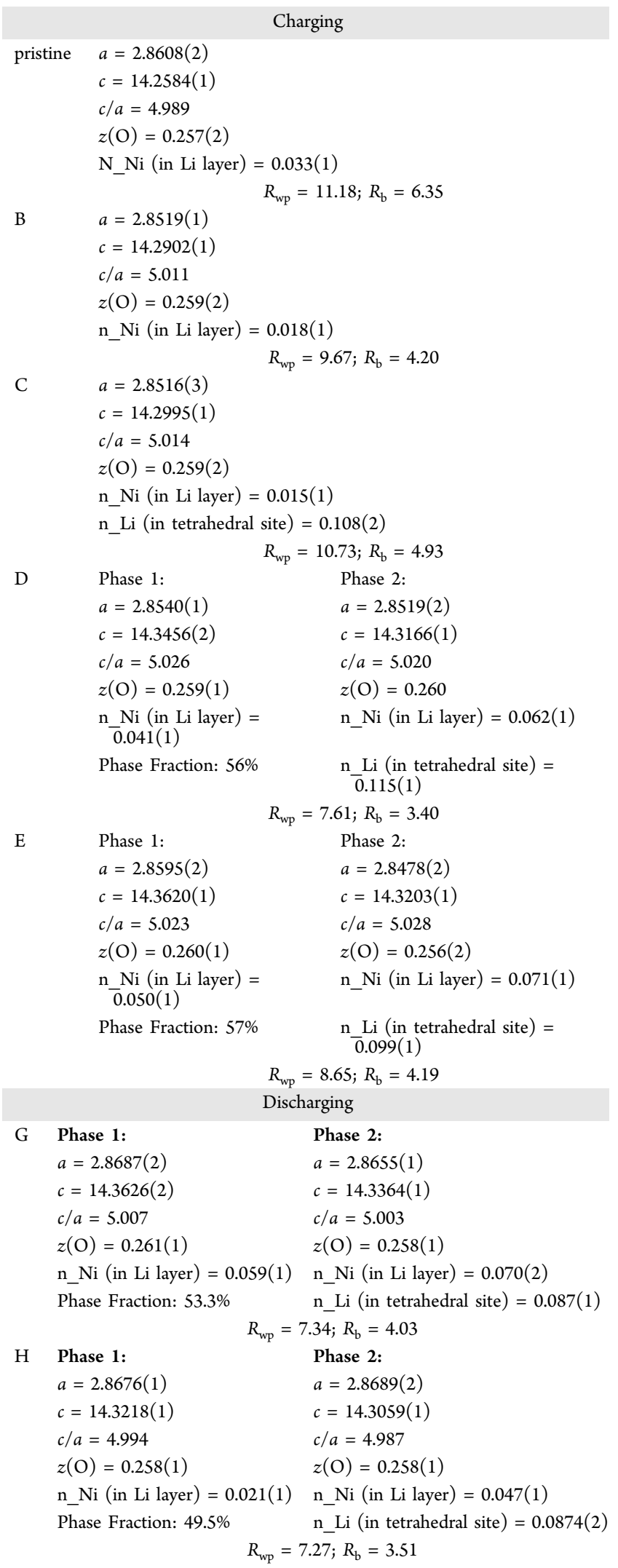


Table 2. Comparison of Rietveld Reliability Factors from Single Layered $R \overline{3} m$ Phase, Single Layered $R \overline{3} m$ Phase with Tetrahedral $\mathrm{Li}^{+}$Occupancy, Two Independent $R \overline{3} m$ Layered Phases with and without Tetrahedral $\mathrm{Li}^{+}$Occupancy

\begin{tabular}{|c|c|c|c|c|c|c|c|c|}
\hline \multirow[b]{2}{*}{ sample name } & \multicolumn{2}{|c|}{$\begin{array}{l}\text { single phase without tetrahedral } \\
\qquad \mathrm{Li}\end{array}$} & \multicolumn{2}{|c|}{ single phase with tetrahedral $\mathrm{Li}$} & \multicolumn{2}{|c|}{ two phases without tetrahedral $\mathrm{Li}$} & \multicolumn{2}{|c|}{ two phases with tetrahedral $\mathrm{Li}$} \\
\hline & $R_{\mathrm{wp}}$ & $R_{\mathrm{B}}$ & $R_{\mathrm{wp}}$ & $R_{\mathrm{B}}$ & $R_{\mathrm{wp}}$ & $R_{\mathrm{B}}$ & $R_{\mathrm{wp}}$ & $R_{\mathrm{B}}$ \\
\hline B & 9.67 & 4.20 & 9.73 & 4.42 & 10.31 & 5.48 & 11.80 & 8.11 \\
\hline $\mathrm{C}$ & 10.73 & 4.95 & 10.70 & 4.93 & 10.17 & 5.21 & 10.21 & 5.21 \\
\hline $\mathrm{D}$ & 10.73 & 4.93 & 8.78 & 3.64 & 7.68 & 3.57 & 7.61 & 3.40 \\
\hline $\mathrm{E}$ & 10.82 & 5.37 & 10.71 & 4.82 & 9.30 & 4.82 & 8.65 & 4.19 \\
\hline G & 9.54 & 6.44 & 9.27 & 5.43 & 7.59 & 5.00 & 7.34 & 4.03 \\
\hline $\mathrm{H}$ & 8.49 & 4.74 & 8.50 & 4.6 & 7.51 & 3.93 & 7.27 & 3.51 \\
\hline
\end{tabular}

At the middle of the plateau region $(4.60 \mathrm{~V}, \mathrm{D}$ in Figure $\mathrm{S} 1)$ the refinement improved when two independent phases, $R \overline{3} \mathrm{~m}$ space groups, were implemented. The addition of a second layered phase with tetrahedral $\mathrm{Li}^{+}$occupancy improved both Rietveld reliability factors $R_{\mathrm{wp}}$ and $R_{\mathrm{B}}$ to 7.61 and 3.40, respectively. The use of a second phase increased the flexibility in the refinement to account for peak broadening as well as peak shoulders in the diffraction pattern (Figure $1 \mathrm{~b}$ ). The refinement results continue to support the evidence of tetrahedral $\mathrm{Li}^{+}$ions forming in the second layered phase during the middle of the first charge cycle.

Lastly, at the end of the first complete charge $(4.80 \mathrm{~V}, \mathrm{E}$ in Figure S1), the XRD pattern shows that the superlattice peaks remain visible (Figure 1a) indicating that long-range cation ordering remains present in the transition metal layer, even after complete delithiation. The addition of a second $R \overline{3} \mathrm{~m}$ phase including tetrahedral $\mathrm{Li}^{+}$ion occupancy improves the pattern fitting and reduces the residual errors of the (003), (104), (110), and (108) peaks over a single layered $R \overline{3} m$ phase or two independent $R \overline{3} m$ layered phases without tetrahedral $\mathrm{Li}^{+}$ occupancy (see Tables 1 and 2). The amount of $\mathrm{Li} / \mathrm{Ni}$ interlayer mixing increased $50 \%$ to 0.05 at the end of the first charge. The increased amount of $\mathrm{Li}^{+}$vacancies increases the likelihood of $\mathrm{Ni}$ ion migration. Previous literature has identified oxygen loss during the plateau region in the Li-excess series of materials. $7,8,14,37$ To explore the possibility of oxygen loss in the structure, oxygen occupancies were refined without constraints. The Rietveld refinement results indicate that in the layered phase with tetrahedral $\mathrm{Li}^{+}$ion formation, $22 \%$ oxygen loss is observed; while the oxygen content of the second layered phase remains constant at 2.0 (see Table 3). Neutron diffraction work is currently in progress to quantify the oxygen vacancy amount more accurately; the result will be reported elsewhere.

The XRD patterns collected during the first electrochemical discharge indicate that the superlattice peaks remain evident until $3.30 \mathrm{~V}$ (position G) as seen in Figure 1a. The superlattice peaks start to fade during the remainder of the discharge cycle, as seen from the XRD pattern following the first full electrochemical cycle, consistent with previous literature. ${ }^{15,25}$ Rietveld refinement of patterns collected during the discharge using a single layered phase no longer leads to pattern convergence and confirms that the material retains the two independent phases with tetrahedral $\mathrm{Li}^{+}$occupancy remaining constant during discharge. Within the states during discharge, the $c$ lattice parameter begins to contract while the $a$ lattice parameter expands, which corresponds to the reduction of $\mathrm{Ni}^{4+}$ to $\mathrm{Ni}^{2+}$.

After the first electrochemical cycle, for the original layered phase, the $c$ lattice parameter increases from the pristine value of $14.2584 \AA$ to $14.3218 \AA$ following discharge to $2.0 \mathrm{~V}$ (H in
Table 3. Comparison of Fitting Models for Position E Using a Single Phase Model and Two Independent Layered Phase with and without Tetrahedral $\mathrm{Li}^{+}$Occupancy

\begin{tabular}{|c|c|c|c|}
\hline $\begin{array}{l}\text { position } E: \\
\text { single phase }\end{array}$ & $\begin{array}{l}\text { position E: two } \\
\text { layered phases }\end{array}$ & $\begin{array}{l}\text { position E: two } \\
\text { layered phases } \\
\text { and tetrahedral } \mathrm{Li}\end{array}$ & $\begin{array}{l}\text { position E: two layered } \\
\text { phases and tetrahedral } \mathrm{Li} \\
\text { with oxygen vacancies }\end{array}$ \\
\hline$a=2.8485$ & $\begin{array}{l}\text { Phase 1: } \\
a=2.8575\end{array}$ & $\begin{array}{l}\text { Phase 1: } \\
a=2.8595\end{array}$ & $\begin{array}{l}\text { Phase 1: } \\
a=2.8595\end{array}$ \\
\hline$c=14.3263$ & $c=14.3446$ & $c=14.3620$ & $c=14.3620$ \\
\hline$c / a=5.029$ & $c / a=5.020$ & $c / a=5.023$ & $c / a=5.023$ \\
\hline$z(\mathrm{O})=0.260$ & $z(\mathrm{O})=0.260$ & $z(0)=0.260$ & $z(\mathrm{O})=0.260$ \\
\hline $\begin{array}{l}\mathrm{n} \text { _Ni (in } \mathrm{Li} \\
\text { layer) }= \\
0.063\end{array}$ & $\begin{array}{l}\mathrm{n} \text { - Ni (in } \mathrm{Li} \\
\text { layer) }= \\
0.079\end{array}$ & $\begin{array}{l}\mathrm{n}_{\text {layer) }} \mathrm{Ni}(\text { in } \mathrm{Li} \\
\text { la.050 }\end{array}$ & $\begin{array}{l}\mathrm{n} \_\mathrm{Ni}(\text { in } \mathrm{Li} \text { layer }) \\
0.050\end{array}$ \\
\hline \multirow[t]{11}{*}{$\begin{array}{c}R_{\mathrm{wp}}=10.71 \\
R_{\mathrm{b}}=4.82\end{array}$} & $\begin{array}{l}\text { Phase Fraction: } \\
57 \%\end{array}$ & $\begin{array}{l}\text { Phase Fraction: } \\
57 \%\end{array}$ & $\mathrm{n} \_$Oxygen $=2.00$ \\
\hline & & & Phase Fraction: 57\% \\
\hline & Phase 2: & Phase 2: & Phase 2: \\
\hline & $a=2.8476$ & $a=2.8478$ & $a=2.8478$ \\
\hline & $c=14.3217$ & $c=14.3203$ & $c=14.3203$ \\
\hline & $c / a=5.029$ & $c / a=5.028$ & $c / a=5.028$ \\
\hline & $z(\mathrm{O})=0.260$ & $z(\mathrm{O})=0.259$ & $z(\mathrm{O})=0.260$ \\
\hline & $\begin{array}{l}\mathrm{n}_{\text {_ }} \mathrm{Ni}(\text { in } \mathrm{Li} \\
\text { layer) }= \\
0.053\end{array}$ & $\begin{array}{l}\mathrm{n}_{-} \mathrm{Ni}(\text { in } \mathrm{Li} \\
\text { layer) }=0.071\end{array}$ & $\underset{0.075}{\mathrm{n} \_\mathrm{Ni}(\text { in Li layer })}=$ \\
\hline & $\begin{array}{r}R_{\text {wp }}=9.30 \\
R_{\mathrm{b}}=4.82\end{array}$ & $\begin{array}{l}\mathrm{n}_{-} \mathrm{Li} \text { (in } \\
\text { tetrahedral } \\
\text { site) }=0.099\end{array}$ & $\begin{array}{l}\mathrm{n}_{-} \mathrm{Li}(\text { in tetrahedral site }) \\
=0.102\end{array}$ \\
\hline & & $\begin{array}{r}R_{\mathrm{wp}}=8.65 \\
R_{\mathrm{b}}=4.19\end{array}$ & n_Oxygen $=1.56$ \\
\hline & & & $R_{\mathrm{wp}}=8.52 ; R_{\mathrm{b}}=3.80$ \\
\hline
\end{tabular}

Figure S1). Moreover, the a lattice parameter expands from $2.8608 \AA$ to $2.8676 \AA$ following complete discharge. The expanded lattice parameters following discharge are consistent with previous research. ${ }^{8}$

3.2. Strain Effects During Electrochemical Cycling. Microstrain effects were extracted by examining the line broadening observed in the XRD patterns obtained during the first electrochemical cycle. From our SEM images, the average particle size does not change much during the first electrochemical cycle, that is, it remains to be above $100 \mathrm{~nm}$. XRD peak broadening contributed from size effect can be excluded. Figure 2 shows microstrain values obtained at different states of charge during the first electrochemical cycle. Williamson-Hall type microstrain can be explained by nonuniform strain effects originating from systematic shifts of atoms from their ideal positions resulting from defects such as point defects, site-disorder, and vacancies as well as plastic deformation (see S5 for more details). ${ }^{38-40}$ The plot shows that the microstrain remains constant from the pristine material through the sloping region to $4.45 \mathrm{~V}$. At the end of the first charge $(\mathrm{E})$ the microstrain increases by $0.1 \%$, which doubles 

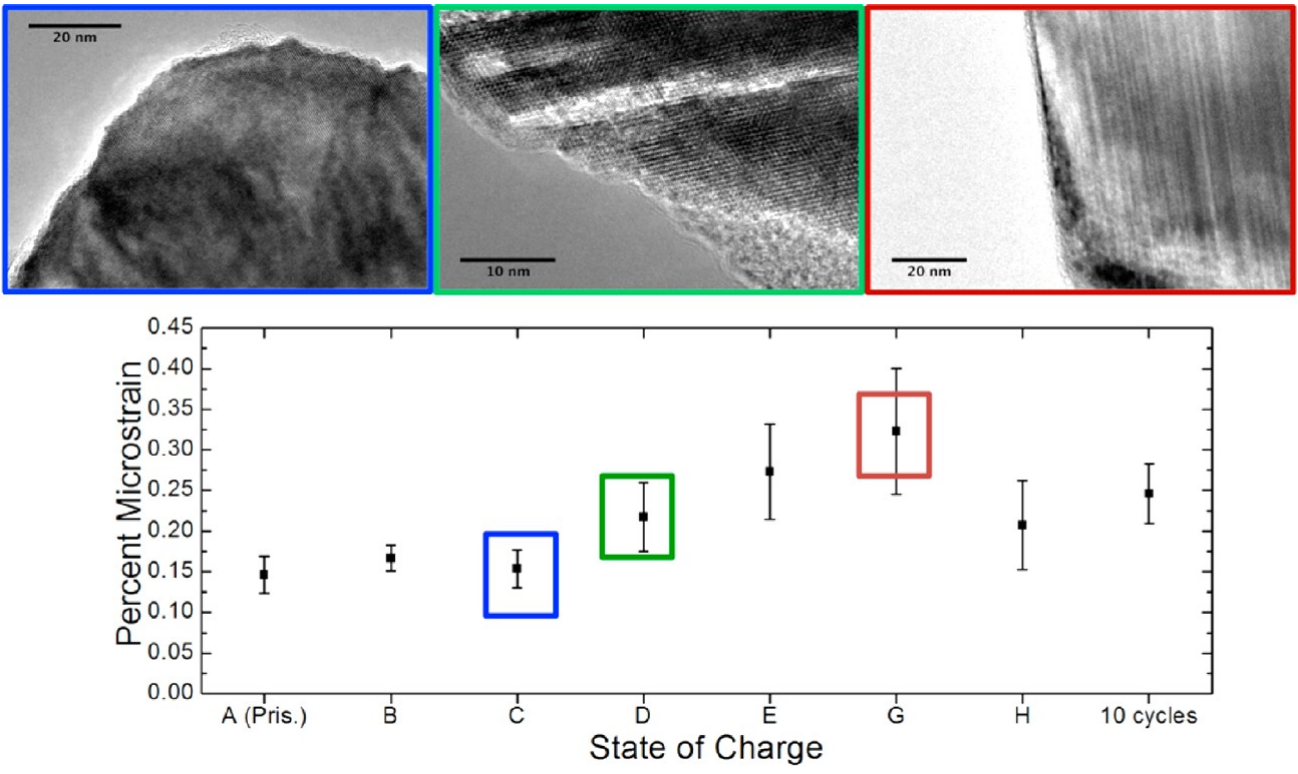

Figure 2. Change in the percent microstrain of $\mathrm{Li}\left[\mathrm{Li}_{1 / 5} \mathrm{Ni}_{1 / 5} \mathrm{Mn}_{3 / 5}\right] \mathrm{O}_{2}$ plotted corresponding to the state of charge during the first electrochemical cycle. Above are TEM images of the highlighted points illustrating increased strain. Error bars shown correspond to $\sigma$.

(a)

(b)

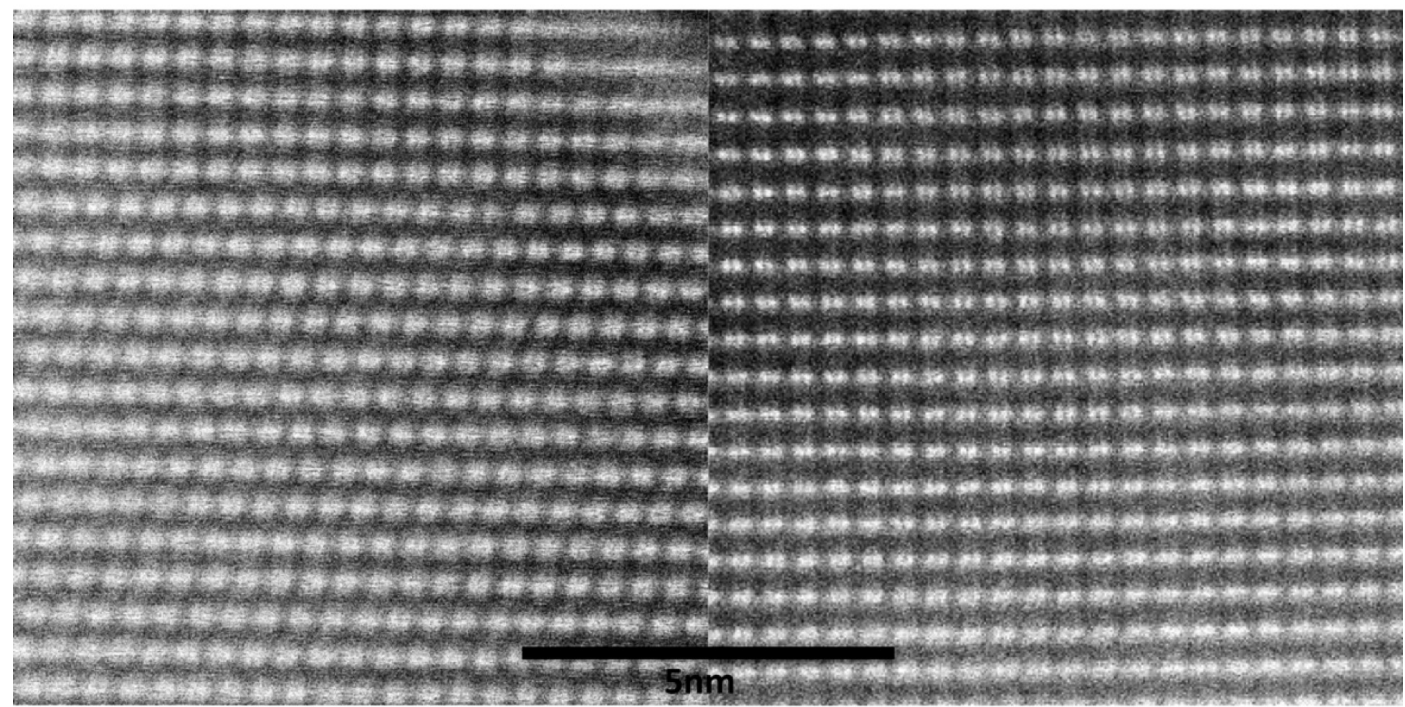

Figure 3. High resolution STEM images of (a) pristine and (b) after 10 cycles.

comparing to the pristine value. However, the errors associated with the microstrain values with states of charge at $4.60 \mathrm{~V}, 4.80$ $\mathrm{V}$, and $3.30 \mathrm{~V}$ have increased significantly. TEM images taken at these states of charge show a high degree of stacking faults and the formation of dislocations within the material. An improvement to the fitting can be applied using the modified Williamson-Hall plot, ${ }^{40-42}$ which will be explored in future studies. The microstrain increase correlates well with the amount of $\mathrm{Li}^{+}$ion vacancies in the delithiated sample as well as site-disordering from the possibility of tetrahedral $\mathrm{Li}^{+}$site formation. Microstrain effects may also originate from cation migration accompanied by oxygen vacancy, $\mathrm{Li} / \mathrm{Ni}$ site mixing, and a second layered phase formation, which are confirmed from Rietveld refinement of the XRD patterns. The microstrain values continue to increase during the first half of the discharge cycle. At this point, $\mathrm{Li}^{+}$ions are re-entering the structure, and the microstrain decreases as additional $\mathrm{Li}^{+}$are intercalated into the material at the end of the discharge cycle. We propose that the decrease in microstrain is a result of oxygen ions re-entering the structure, which is evidenced by changes in both intensity and position of our EELS oxygen pre-edge and K-edge data. The microstrain generated during the first electrochemical cycle does not completely recover to the pristine material value, indicating irreversible changes during the first cycle, which may contribute to the irreversible capacity loss.

3.3. TEM/STEM. Figure 2 depicts images at three different points along the first electrochemical cycle (C, D, and G points). Multiple grains were selected for study, and the results are consistent, therefore only representative data are shown here. It has been found by previous studies that the pristine material shows well faceted surfaces, a high degree of crystallinity, and well layered properties in the bulk that extend to the surface. ${ }^{5}$ The TEM image following charging to the end of the sloping region at $4.45 \mathrm{~V}$ (C) illustrates the formation of 

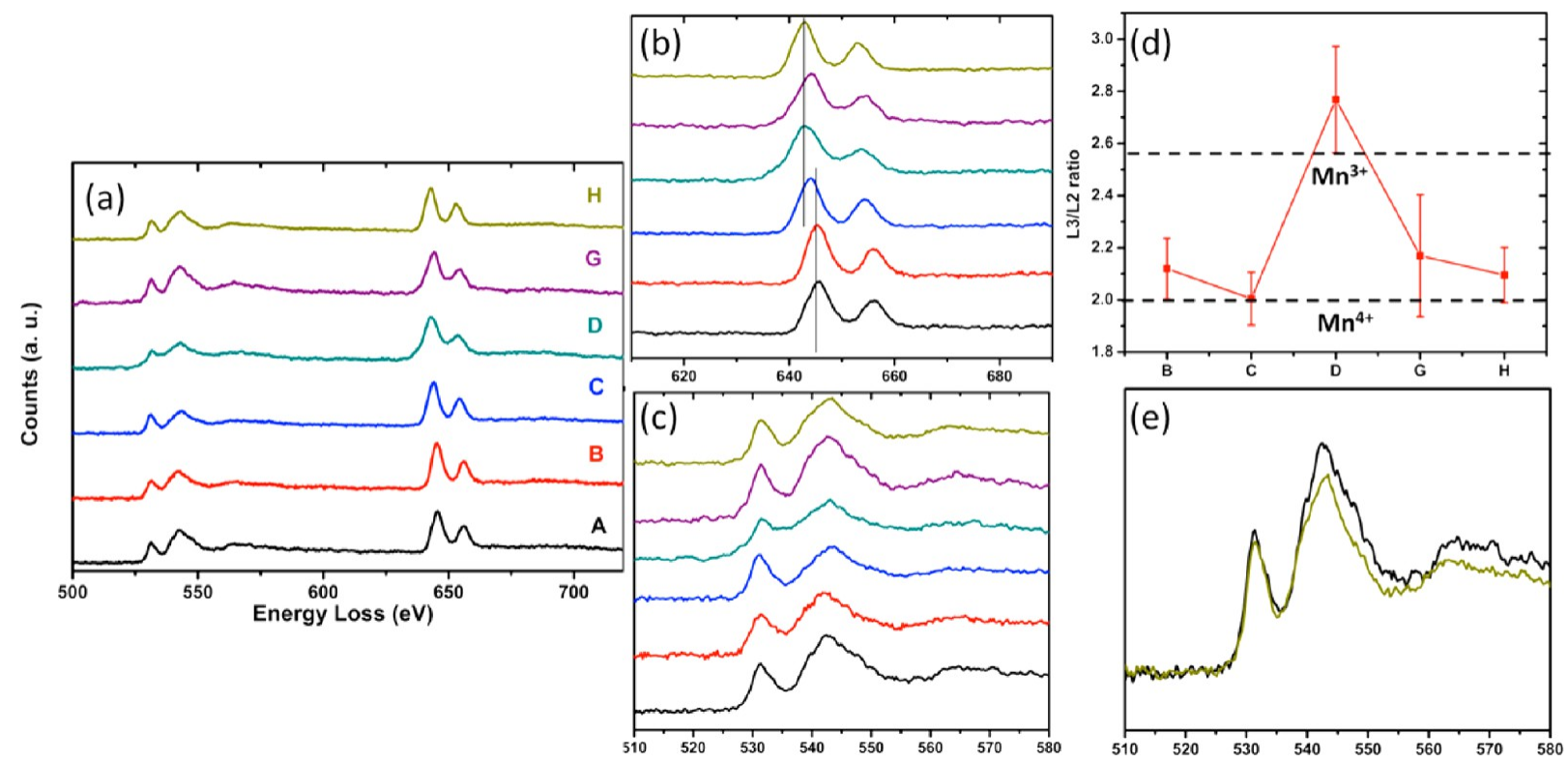

Figure 4. Representative EELS spectra of Mn L-edge and $\mathrm{O}$ K-edge from the bulk of the $\mathrm{Li}\left[\mathrm{Ni}_{1 / 5} \mathrm{Li}_{1 / 5} \mathrm{Mn}_{3 / 5}\right] \mathrm{O}_{2}$ during the first electrochemical charging and discharging cycles. (a) Overall EELS spectrum; (b) Mn L-edge; (c) O K-edge; (d) $\mathrm{Mn} \mathrm{L}_{3} / \mathrm{L}_{2}$ ratio; (e) O K-edge comparison of pristine and after one cycle.

nanocracking extending through the first $20 \mathrm{~nm}$ of the material. The figure shows that the faceted surfaces in the pristine material begin to become less clearly defined which may be an indication of material loss. Attempts to capture HAADF-STEM images were unsuccessful from the beginning of the voltage plateau region throughout the remainder of the first electrochemical charge/discharge cycle because the evolution of strains and defects prevented visualization of the atomic columns. Blue-framed low magnification TEM images taken at the middle of the plateau region $(4.60 \mathrm{~V}, \mathrm{D})$ correspond to a charging capacity of $200 \mathrm{mAh} / \mathrm{g}$. The figure shows a significant increase in the formation of nanocracks that span into the bulk of the material.

Upon discharge to $3.30 \mathrm{~V}(\mathrm{G})$, approximately $0.5 \mathrm{Li}^{+}$ions are reintercalated into the structure. The TEM image in the maroon frame suggests that the amount of stacking faults and defects continue to increase in the material. The electron diffraction pattern of the particle reveals that there are no distinct spots associated with a well layered structure, but streaking indicates changes in the long-range ordering and layeredness of the material (Figure S6). This streaking is consistent with the large amount of microstrain observed by XRD. Following discharge to $2.00 \mathrm{~V}(\mathrm{H})$, the nanocracks as well as the defects seen at previous points during the electrochemical cycle are not commonly observed anymore in most of the particles, which enables the HAADF-STEM imaging of the material. The image shows that the bulk of the material still maintains the well-layered structure; however, the contrast within the first $2 \mathrm{~nm}$ of the surface changes. Along the $(00 \overline{1})$ direction, the dark columns become much brighter on the surface. ${ }^{43}$ The contrast matches that of the neighboring TM columns. This suggests that, following the first electrochemical cycle, a second phase has formed, consistent with previous findings. ${ }^{26}$ Figure 3 compares the HAADF-STEM images from the pristine and after 10 electrochemical cycles recorded along the $[\overline{11} 0]$ zone axis. The stacking sequence of the layers in the pristine material completely changed after 10 electrochemical cycles. In the pristine material, there is evidence of stacking faults; however, following electrochemical cycling, the material adopts a more uniform structure. This may suggest that the oxygen framework tends to adopt a different stacking after the oxygen vacancy formation and cation migration, and such stacking changes may be responsible for the disappearance of the superstructure peaks in the XRD pattern.

3.4. EELS. Figure $4 a$ compares the EELS spectra of the oxygen K-edge and manganese L-edges from the bulk of the structure taken at different states during electrochemical cycling. The structural evolution during the first electrochemical cycle can be interpreted using changes in the onset energy and the fine structures in the spectra. The intensities of all the spectra are normalized to the highest intensity peak. The onset energy of $\mathrm{O}$ K-edge prepeak is aligned to $532 \mathrm{eV}$. Therefore, our analysis of the $\mathrm{O}$ K-edge is limited to the fine structures and not the chemical shift of $\mathrm{O}$ K-edge. The $\mathrm{L}_{3}$ and $\mathrm{L}_{2}$ of transition metals are due to the transition from $2 \mathrm{p}^{3 / 2}$ to $3 \mathrm{~d}^{3 / 2}$ and $3 \mathrm{~d}^{5 / 2}$ and from $2 \mathrm{p}^{1 / 2}$ to $3 \mathrm{~d}^{3 / 2}$, respectively. Their intensities are correlated to the unoccupied bands in $3 \mathrm{~d}$ orbitals. Previous studies have shown that the $\mathrm{L}_{3} / \mathrm{L}_{2}$ ratio is sensitive to the valence state of $\mathrm{Mn}^{44,45}$

Examining the Mn L-edge onset energy during the sloping region of the first charging cycle shows that the peak shifts to a lower energy loss beginning at position $C$ (Figure $4 \mathrm{~b}$ ) which indicates a lower oxidation state. Analysis of the $\mathrm{L}_{3} / \mathrm{L}_{2}$ edge ratio (shown in Figure 4d) further supports the change in the $\mathrm{Mn}$ oxidation state to a value at position C. During the discharge, the $\mathrm{Mn}$ valence state returns back to $4+$ following the first electrochemical cycle. The onset energy for the $\mathrm{Mn} \mathrm{L}_{3}$ peak remaining shifted to lower energies following discharge may signify a changing local atomic arrangement.

Figure $4 \mathrm{c}$ shows the oxygen K-edge spectra. The splitting into two peaks for the $\mathrm{O}$ K-edge is a characteristic of the layered material. In the layered material, oxygen forms an $\mathrm{O} 3$ framework and the TM resides in octahedral sites. The crystal field of the TM splits into three $t_{2 g}$ orbitals at a lower energy and two $e_{g}$ orbitals at a higher energy level. The K-edge of oxygen is the consequence of the transition of $1 \mathrm{~s}$ electrons to 
the unoccupied $2 \mathrm{p}$ orbitals, which hybridized with the TM $3 \mathrm{~d}$ orbitals. The splitting of the $\mathrm{O}$ K-edge corresponds to the splitting of the TM $3 \mathrm{~d}$ orbitals.

In positions $\mathrm{C}$ and $\mathrm{D}$, the ratio of the first peak to the second peak increases, which may result from a larger amount of unoccupied $t_{2 g}$ orbitals and a change in local environment of oxygen, such as bond length, oxygen vacancy formation, etc. The increasing ratio can be from the oxidation of $\mathrm{TM}$, e.g., $\mathrm{Ni}^{2+}$ to $\mathrm{Ni}^{4+}$. The energy difference in the two peaks can also be an indication of the oxygen local environment change. From position $\mathrm{C}$ through the end of the first cycle, the difference in the two oxygen peaks is the same, which is larger than positions $\mathrm{A}$ and $\mathrm{B}$. This may be a result from oxygen vacancy formation as well as stronger bonding between oxygen and TM, which is not reversible at the end of the first cycle. These results show that, in the bulk structure beginning at the voltage plateau region, the local environments of $\mathrm{Ni}^{4+}, \mathrm{Mn}^{4+}$, and oxygen are simultaneously changing and may all participate in charge compensation during the voltage plateau region, some of which is not reversible.

\section{DISCUSSION}

4.1. Source of Anomalous Capacity-Oxygen Activation. Previous studies of the Li-excess series of layered materials have identified the oxygen activation mechanism as the source for the anomalous capacity. ${ }^{7,8,14,46}$ The present research uses the combined results obtained from SXRD, TEM, and EELS at various states of charge to further explain the source of anomalous capacity identified within this series of materials. Rietveld refinement of SXRD patterns following the first charging cycle ( $\mathrm{E}$ in Figure 1C) identified the loss of approximately $20 \%$ oxygen from the bulk structure within one of the two layered phases refined. The amount of oxygen loss to accommodate the removal of the additional $0.6 \mathrm{~mol} \mathrm{Li}^{+}$ions corresponds to $0.3 \mathrm{~mol}$ (15\%), assuming the contribution of 2 electrons. Recent reports have used refinement to identify the loss of structural oxygen; however, only less than half of the theoretically proposed oxygen loss was found. ${ }^{7}$ A possible explanation for the smaller than expected oxygen loss is that the transition metal ions are also contributing to the anomalous capacity. Analysis of the Mn oxidation state from the EELS reveals a change from 4+ to nearly $3+$ then returns to 4+ during the discharge cycle. It is the first identification of the changing Mn oxidation state within this series of materials during the first electrochemical cycle. The changing Mn oxidation state leads to a shift in the oxygen octahedral positions, which can cause local distortions in the lattice causing increased peak broadening as evidenced in the elevated microstrain values from the Williamson-Hall plots at position E. $\mathrm{Mn}^{3+}$ may also go through disproportionation reaction, which may contribute to the irreversible capacity during the first cycle. Further evidence of changing oxygen local environment is evidenced through our EELS data.

4.2. Structural Changes Caused by Oxygen Vacancies. During the initial electrochemical charging before the voltage plateau region, structural changes observed through Rietveld refinement of XRD patterns and the TEM images are minimal. The amount of $\mathrm{Li} / \mathrm{Ni}$ interlayer mixing decreases, indicating that the layered properties of the material are improving. Rietveld refinement indicates that neither the tetrahedral $\mathrm{Li}^{+}$ ions nor the second layered phase forms within this region. When the electrochemical charging cycle reaches the voltage plateau, at position $\mathrm{D}$, there is some evidence of a second phase along with tetrahedral $\mathrm{Li}^{+}$ion formation. EELS spectra indicate a possibility of oxygen vacancy formation at the beginning of the plateau region, and Rietveld refinement confirms the oxygen loss following the plateau region. We propose that these oxygen vacancies and the distortion in the oxygen octahedral create stacking faults within the lattice seen in the TEM micrographs (Figure 2). It has been previously reported that ordered and disordered vacancies, such as vacant $\mathrm{Li}^{+}$and $\mathrm{O}^{2-}$ sites, as well as the creation of stacking faults are driven by transition metal or lithium layer gliding to reduce the structural free energy. ${ }^{47,48}$ The layer gliding provides a mechanism for the formation of domain structure and second phase formation, which result in different stacking sequences of the crystal structure (Figure 3). This second phase is created during the plateau region, and Rietveld refinement confirms the presence throughout the remainder of the first electrochemical cycle. The TEM micrographs show that at the beginning of the discharge, a large amount of stacking faults are evident.

With the introduction of the stacking faults and the corresponding increase in microstrain within the material, the cations within $\mathrm{Li}\left[\mathrm{Li}_{1 / 5} \mathrm{Ni}_{1 / 5} \mathrm{Mn}_{3 / 5}\right] \mathrm{O}_{2}$ migrate and undergo significant electronic state changes. At the beginning of the plateau region the $\mathrm{Ni}$ and $\mathrm{Mn}$ ions are completely oxidized to the $4+$ states and the amount of $\mathrm{Li} / \mathrm{Ni}$ interlayer mixing remains below 0.02 . Within the plateau region, the first evidence of tetrahedral $\mathrm{Li}^{+}$is identified, indicating favorable cation migration environments. The vacancies created in the oxygen layers not only induce the stacking faults but also enable $\mathrm{Li} / \mathrm{Ni}$ cation migration by hopping through nearby vacancies. During states of charge within the voltage plateau, the $\mathrm{Li} / \mathrm{Ni}$ interlayer mixing increases to a maximum mixing of 0.071 at the end of the first charge cycle and remains elevated at the initial stages of the discharge cycle. Furthermore, electronic state changes occur in the Mn ions. The EELS spectra reveal a change in the energy loss to a lower position and detailed analysis of the $L_{3} / L_{2}$ peak intensity ratio shows evidence of $\mathrm{Mn}^{3+}$ formation. Both of these changes show that not only does the oxygen participate in the electrochemical compensation during the voltage plateau, but that TM also contributes. The stacking faults generated during electrochemical cycling contributed to the microstrain seen during the first electrochemical cycle. These factors prevented high resolution structural imaging at the high state of charge (point E); however, following discharging to $2.0 \mathrm{~V}$ the structure has reversibly reconfigured to a model closer to the pristine material where the $\mathrm{Mn}$ oxidation in the bulk is almost fully back to $4+$ and the $\mathrm{Ni}$ ion has reduced to $2+$.

It is clear that permanent structural rearrangements have occurred. There is permanent lattice expansion in both the $a$ and $c$ lattice parameters as well as the formation of tetrahedral $\mathrm{Li}^{+}$ions and a second layered phase, as well as the change in the layer stacking in the original layered phase. These permanent structural modifications become more evident and the phase percentages increase as the material continues electrochemically cycling as we have shown previously. ${ }^{26}$ Moreover, following the first electrochemical cycle, the STEM image clearly shows the well-layered structure in the bulk; however, the surface structure of the cycled grain has changed to a defect spinel phase. Identifying how this material continues to evolve during long-term cycling is a critical piece necessary to identify the source of capacity fading as well as the depression in discharge voltage in this class of compounds. 


\section{CONCLUSION}

Based on the experimental results presented, we have shown that the mechanisms providing charge compensation during the first electrochemical cycle of the Li-excess layered oxide materials are complex. We have presented direct evidence to describe the lithium deintercalation mechanisms at different states of charge. Oxygen vacancies forming and possible oxygen activation within the bulk structure are identified through EELS and Rietveld refinement of SXRD. The evidence indicates that the oxygen activation may be responsible for the formation of stacking fault defects as well as for facilitating cation migration, including Ni migration to the $\mathrm{Li}$ layer. These defects created within the structure lead to the increase of significant microstrains observed within the bulk structure. The microstrain continually increases during the voltage plateau and into the beginning of the discharge cycle where the largest amount of cation mixing, oxygen vacancies, and lattice parameter expansion are observed.

Our results presented here suggest that a combination of these mechanisms during the first electrochemical cycle, particularly in the voltage plateau region, impede lithium diffusion, which may contribute to the low intrinsic rate capabilities and large irreversible capacity losses of this material. Identification of these mechanisms forming during the first electrochemical cycle raises questions about how surface coatings improve irreversible capacity fading and whether they remove or change these dynamic processes during the first electrochemical cycle. The present research also raises questions about the continually changing nature of these electrodes upon cycling. Only through continued research using sophisticated instrumentations such as atomic resolution STEM and EELS as well as high resolution synchrotron X-ray diffraction and neutron diffraction will the complex nature of the interactions in the Li-excess layered transition metal oxides be uncovered.

\section{ASSOCIATED CONTENT}

\section{S Supporting Information}

Electrochemical voltage profile of first cycle (S1). State of charge, voltages, and capacities summary of different points (S2). Profile fits for Rietveld refinement of different points (S3). X-ray absorption spectroscopy (S4). The Williamson-Hall method (S5). Electron diffraction pattern (S6). This material is available free of charge via the Internet at http://pubs.acs.org

\section{AUTHOR INFORMATION}

\section{Corresponding Author}

*E-mail: shirleymeng@ucsd.edu.

\section{Present Address}

"Global Technology \& Innovation, Power Solutions, Johnson Controls, Milwaukee, WI 53209, USA.

\section{Notes}

The authors declare no competing financial interest.

\section{ACKNOWLEDGMENTS}

UCSD work was supported by the Assistant Secretary for Energy Efficiency and Renewable Energy, Office of Vehicle Technologies of the U.S. Department of Energy under Contract No. DE-AC02-05CH11231, Subcontract No. 7056412 under the Batteries for Advanced Transportation Technologies (BATT) Program. C.R.F. acknowledges the financial support from Florida Energy System Consortium through University of Florida under Award No. 80859. D.Q. would like to acknowledge Dr. Huolin Xin for providing the scripts for $\mathrm{L}_{3} / \mathrm{L}_{2}$ analysis. The synchrotron X-ray diffraction patterns were collected at Argonne National Laboratory on beamline 11-BM through the general user proposal mail-in program (GUP-13210). a-S/TEM and EELS analysis is carried out at the ORNL Shared Research Equipment (SHaRE) User Facility, which is sponsored by the Office of Basic Energy Sciences, U.S. Department of Energy. The authors acknowledge Dr. Gabriel Veith and Dr. Nancy Dudney for assistance and Dr. Bo $\mathrm{Xu}$ and Dr. M. Yang for their valuable discussions.

\section{REFERENCES}

(1) http://www.mineralprices.com/ (2012).

(2) Boulineau, A.; Simonin, L.; Colin, J.-F.; Canévet, E.; Daniel, L.; Patoux, S. Chem. Mater. 2012, 24, 3558.

(3) Thackeray, M. M.; Kang, S.-H.; Johnson, C. S.; Vaughey, J. T.; Benedek, R.; Hackney, S. A. J. Mater. Chem. 2007, 17, 3112.

(4) Meng, Y. S.; Ceder, G.; Grey, C. P.; Yoon, W. S.; Jiang, M.; Breger, J.; Shao-Horn, Y. Chem. Mater. 2005, 17, 2386.

(5) Lei, C. H.; Bareno, J.; Wen, J. G.; Petrov, I.; Kang, S. H.; Abraham, D. P. J. Power Sources 2008, 178, 422.

(6) Yoon, W. S.; Paik, Y.; Yang, X. Q.; Balasubramanian, M.; McBreen, J.; Grey, C. P. Electrochem. Solid-State Lett. 2002, 5, A263.

(7) Yabuuchi, N.; Yoshii, K.; Myung, S.-T.; Nakai, I.; Komaba, S. J. Am. Chem. Soc. 2011, 133, 4404.

(8) Lu, Z.; Dahn, J. R. J. Electrochem. Soc. 2002, 149, A815.

(9) Robertson, A. D.; Bruce, P. G. Electrochem. Solid-State Lett. 2004, 7, A294.

(10) Park, Y. J.; Hong, Y.-S.; Wu, X.; Ryu, K. S.; Chang, S. H. J. Power Sources 2004, 129, 288.

(11) Fell, C. R.; Carroll, K. J.; Chi, M.; Meng, Y. S. J. Electrochem. Soc. 2010, 157, A1202.

(12) Li, J.; Klöpsch, R.; Stan, M. C.; Nowak, S.; Kunze, M.; Winter, M.; Passerini, S. J. Power Sources 2011, 196, 4821.

(13) Kang, S. H.; Thackeray, M. M. J. Electrochem. Soc. 2008, 155, A269.

(14) Armstrong, A. R.; Holzapfel, M.; Novak, P.; Johnson, C. S.; Kang, S. H.; Thackeray, M. M.; Bruce, P. G. J. Am. Chem. Soc. 2006, $128,8694$.

(15) Tran, N.; Croguennec, L.; Menetrier, M.; Weill, F.; Biensan, P.; Jordy, C.; Delmas, C. Chem. Mater. 2008, 20, 4815.

(16) De Souza, R. A.; Ramadan, A.; Horner, S. Energy Environ. Sci. 2012, 5, 5445.

(17) Kushima, A.; Yildiz, B. J. Mater. Chem. 2010, 20, 4809.

(18) Araki, W.; Imai, Y.; Adachi, T. J. Eur. Ceram. Soc. 2009, 29, 2275.

(19) Kilner, J. A. Nat. Mater. 2008, 7, 838.

(20) Mavrikakis, M.; Hammer, B.; Nørskov, J. K. Phys. Rev. Lett. 1998, 81, 2819.

(21) Maier, J. Nat. Mater. 2005, 4, 805.

(22) Blamire, M. G.; MacManus-Driscoll, J. L.; Mathur, N. D.; Barber, Z. H. Adv. Mater. 2009, 21, 3827.

(23) Schichtel, N.; Korte, C.; Hesse, D.; Janek, J. Phys. Chem. Chem. Phys. 2009, 11, 3043.

(24) Garcia-Barriocanal, J.; Rivera-Calzada, A.; Varela, M.; Sefrioui, Z.; Iborra, E.; Leon, C.; Pennycook, S. J.; Santamaria, J. Science 2008, $321,676$.

(25) Jiang, M.; Key, B.; Meng, Y. S.; Grey, C. P. Chem. Mater. 2009, 21, 2733.

(26) Xu, B.; Fell, C. R.; Chi, M.; Meng, Y. S. Energy Environ. Sci. 2011, 4, 2223.

(27) Ito, A.; Shoda, K.; Sato, Y.; Hatano, M.; Horie, H.; Ohsawa, Y. J. Power Sources 2011, 196, 4785.

(28) Rodríguez-Carvajal, J. In Satellite Meeting on Powder Diffraction of the XV Congress of the IUCr Toulouse, France, 1990; p 127.

(29) Larson, A. C.; Dreele, R. B. V. General Structure Analysis System (GSAS); Los Alamos National Laboratory Report (LAUR) 86-748; Los Alamos National Laboratory: 2004. 
(30) Toby, B. J. Appl. Crystallogr. 2001, 34, 210.

(31) Thompson, P.; Cox, D. E.; Hastings, J. B. J. Appl. Crystallogr. $1987,20,79$.

(32) Finger, L. W.; Cox, D. E.; Jephcoat, A. P. J. Appl. Crystallogr. 1994, 27, 892.

(33) Pennycook, S. J. Ultramicroscopy 1989, 30, 58.

(34) James, E. M.; Browning, N. D. Ultramicroscopy 1999, 78, 125.

(35) Wang, Z. L.; Yin, J. S.; Jiang, Y. D. Micron 2000, 31, 571.

(36) Shannon, R. D.; Prewitt, C. T. Acta Crystallogr. 1969, B25, 925.

(37) Wu, Y.; Murugan, A. V.; Manthiram, A. J. Electrochem. Soc. 2008, 155, A635.

(38) Stephens, P. J. Appl. Crystallogr. 1999, 32, 281.

(39) Williamson, G. K.; Hall, W. H. Acta Metallurg. 1953, 1, 22.

(40) Groma, I.; Ungar, T.; Wilkens, M. J. Appl. Crystallogr. 1988, 21, 47.

(41) Ungar, T.; Borbely, A. Appl. Phys. Lett. 1996, 69, 3173.

(42) Krivoglaz, M. A.; Ryboshapka, K. P. Phys. Met. Metallogr. 1963, $15,18$.

(43) Carroll, K. J.; Qian, D.; Fell, C. R.; Veith, G. M.; Chi, M.; Dudney, N. J.; Meng, Y. S. Energy Environ. Sci. 2012, submitted.

(44) Kurata, H.; Colliex, C. Phys. Rev. B 1993, 48, 2102.

(45) Riedl, T.; Gemming, T.; Wetzig, K. Ultramicroscopy 2006, 106, 284.

(46) Yu, D. Y. W.; Yanagida, K.; Kato, Y.; Nakamura, H. J. Electrochem. Soc. 2009, 156, A417.

(47) Paulsen, J. M.; Thomas, C. L.; Dahn, J. R. J. Electrochem. Soc. 1999, 146, 3560.

(48) Robertson, A. D.; Armstrong, A. R.; Bruce, P. G. Chem. Mater. 2001, 13, 2380. 